\title{
Repeatability and Reproducibility of a Quantitative Assessment of the Retinal Vascular Network Fractal Dimension Measured With Swept-source Optical Coherence Tomography Angiography in Healthy Eyes
}

\section{Déa Haddad}

Centre Hospitalier Regional Universitaire De Dijon

Louis Arnould ( $\nabla$ louis.arnould@chu-dijon.fr)

Centre Hospitalier Regional Universitaire De Dijon

Florian Baudin

Centre Hospitalier Regional Universitaire De Dijon

Pierre-Henry Gabrielle

Centre Hospitalier Regional Universitaire De Dijon

Marc Sarossy

RMIT University

Alain M. Bron

Centre Hospitalier Regional Universitaire De Dijon

Behzad Aliahmad

RMIT University

Catherine Creuzot-Garcher

Centre Hospitalier Regional Universitaire De Dijon

\section{Research Article}

Keywords: Fractal dimension, Healthy volunteers, Swept-source OCT angiography, Superficial retinal capillary plexus, Deep retinal capillary plexus

Posted Date: February 26th, 2021

DOI: https://doi.org/10.21203/rs.3.rs-244652/v1

License: (c) (1) This work is licensed under a Creative Commons Attribution 4.0 International License.

Read Full License 


\section{Abstract}

Background: To evaluate the repeatability and reproducibility of the retinal vascular network fractal dimension (FD) measured from swept-source optical coherence tomography angiography (SS-OCTA) images of healthy eyes.

Methods: A cross-sectional study was undertaken with young healthy volunteers who had no reported cardiac risk factors or ocular disease history. For each participant, three SS-OCTA images $(12 \times 12 \mathrm{~mm})$ were acquired using the Cirrus Plex Elite 9000 (Carl Zeiss Meditec AG, Jena, Germany) by two ophthalmologists. The data from one eye per participant were retained for analysis. Automated segmentation was obtained from both the superficial and deep capillary plexuses. Fractal box-counting analyses were performed. The intraclass correlation coefficients (ICC) and the coefficient of variation (CV) were used as measures for repeatability and reproducibility.

Results: A total of 43 eyes of healthy volunteers were included. The mean \pm standard deviation (SD) age was $30 \pm 6.2$ years. The mean retinal vascular network FD was 1.693 in the superficial and 1.694 in the deep vascular layer in our healthy cohort. For repeatability, the ICC was $0.722(95 \% \mathrm{Cl}, 0.541-0.839)$ in the superficial capillary plexus and $0.828(95 \% \mathrm{Cl}, 0.705-0.903)$ in the deep capillary plexus. For reproducibility, the ICC was $0.651(95 \% \mathrm{Cl}, 0.439-0.795)$ and $0.363(95 \% \mathrm{Cl}, 0.073-0.596)$ at the superficial and deep capillary plexus, respectively. The CV for each observer was less than $5 \%$ in the two plexuses.

Conclusions: In this study, the retinal vascular network FD provided new repeatability and reproducibility quantitative data using SS-OCTA with healthy participants.

\section{Introduction}

Quantitative description of the retinal vascular network may be indicative of the systemic microvascular network and may help predict future cardiovascular and cerebrovascular events [1-6]. Thus, there is growing interest in thorough quantitative measurement of retinal vascular network features. Retinal arteriole narrowing and retinal vein widening on retinal fundus photographs were shown to be associated with increased long-term cardiovascular outcomes [7]. However, the retinal vascular network could provide more information. The advances in retinal imaging and automated image processing have shed new light on associations between more complex retinal vessel metrics such as the fractal dimension (FD) and cardiovascular and cerebrovascular disorders [8]. The retinal vascular network FD is a geometric index of complexity. Mainster was the first to describe the retinal microvascular network as a fractal [9]. The FD of the retinal vascular network has already been reliably measured from fundus photographs [10, 11]. Previous studies based on fundus photography have suggested that this metric may be an effective method to assess early vascular progression of diabetic retinopathy [12] and to identify patients with proliferative diabetic retinopathy [13]. 
Optical coherence tomography angiography (OCTA) may improve description of the retinal vascular network in daily clinical practice. OCTA is a non-invasive imaging technique for assessment of the retinal microcirculation without the use of dye injections $[14,15]$. OCTA makes it possible to study both the superficial capillary plexus (SCP) and the deep capillary plexus (DCP), which cannot be individualised from the images acquired via standard angiography [16]. Recently introduced swept-source optical coherence tomography (SS-OCT) uses a short cavity swept laser with a tunable wavelength of operation instead of the diode laser used in spectral-domain OCT (SD-OCT). SS-OCT offers higher imaging speeds, greater detection efficiency, improved imaging range and deeper penetration with reduced sensitivity rolloff [17]. Currently, it is possible to automatically assess vascular density, vascular perfusion and the foveal avascular zone area by means of SS-OCTA. By contrast, measuring the retinal vascular network FD with SS-OCTA remains complex and there is no normative database available [18]. Previous studies reported high reproducibility for vascular density and foveal avascular zone area with OCTA in healthy volunteers and in patients with retinal diseases [19-21]. We selected the specific metric of FD because it can potentially summarise the retinal microvascular network. Retinal vascular network FD has been widely studied with fundus photography $[9,12,10]$ and SD-OCTA $[22,23]$, but the literature on FD and SSOCTA is sparse. Moreover, the merit of this non-invasive parameter has been proven in various multidisciplinary areas combining ophthalmology with cardiology $[24,25]$, neurology $[8,26]$ and endocrinology $[12,27]$.

The aim of this study was to evaluate the utility of the retinal vascular network FD, by assessing its normal values, and to assess the repeatability and reproducibility of this technique with SS-OCTA images.

\section{Materials And Methods}

\section{Study participants}

We conducted a cross-sectional descriptive study in the department of ophthalmology at Dijon University Hospital, France, between 1 November, 2017, and 1 June, 2018. The protocol was approved by the regional ethics committee and followed the tenets of the Declaration of Helsinki. All patients gave their written informed consent for the examinations before enrolment. We followed the GRRAS (Guidelines for Reporting Reliability and Agreement Studies) [28].

We enrolled consecutive healthy volunteers between 18 and 55 years of age. The participants' demographic data were collected, including age, sex and ocular history. Participants with a self-reported eye disease, cardiovascular history or neurological history were excluded from the analysis. All participants benefited from a comprehensive eye examination and SS-OCTA acquisition on the same day. We also measured blood pressure with an automated device, after 5 min of rest in a seated position. Weight and height were recorded to calculate each participant's body mass index (BMI). Globe axial length was measured using the IOL master 500 (Carl Zeiss Meditec, Jena, Germany). Measurements were taken on both eyes, but the data from one eye only were retained for analysis, according to the following procedure: (1) SS-OCTA of the right eye for participants born in even-numbered years and the left eye for 
those born in odd-numbered years; (2) if a scan was uninterpretable for one eye, the scan of the other eye was retained for analysis; (3) if both eyes had inadequate signal strength $(<7 / 10)$ or significant artefacts on the perfusion map (lines or gap), the participant was excluded.

\section{Image acquisition}

SS-OCTA images were acquired with the Cirrus Plex Elite 9000 (Carl Zeiss Meditec, Jena, Germany), which uses a swept laser source with a central wavelength between $1040 \mathrm{~nm}$ and $1060 \mathrm{~nm}$ and a rate of 100,000 A-scans per second. All participants underwent SS-OCTA examination under mydriasis obtained with tropicamide $0.5 \%$ eyedrops (Thea, Clermont-Ferrand, France). The study participants underwent imaging in a single visit, including three sets of scans. Two independent, trained observers performed the $12 \times 12$-mm region scans (twice, 1 min apart by observers 1 and 2 ) and repeated once by the second examiner 5 min later (observer 2) resulting in three $12 \times 12-\mathrm{mm}$ scans. We investigated both superficial and deep retinal capillaries (Fig. 1), which were automatically segmented by the SS-OCTA system software. We did not make any manual adjustments because we wanted to reproduce the routine followed in daily practice.

\section{Retinal vascular network fractal dimension analysis}

A special form of Mandelbrot's FD known as the binary box-counting dimension was used to analyse SSOCTA images [29]. For this purpose, images were first binarised using a fixed threshold for all images to obtain microvasculature structures in the highest possible contrast from their background, where vessels were shown in white against a black background. The next step involved image skeletonisation and successive thinning of the vasculature until each vessel was presented by its centre line with a thickness of only one pixel. All images were automatically examined to ensure no discontinuities were introduced to the vessel structure due to the binarisation and skeletonisation process.

To obtain the box-counting FD, the skeletonised images were superimposed on a grid of squares and the total number of boxes (squares) containing at least one pixel of the structure were counted. This process was repeated continuously by reducing the mesh size in each step (increasing the total number of meshes covering the entire image). All data points (total number of meshes counted in each step vs mesh size) were plotted on a log-log plot and the slope of the line with the best fit to the data was taken as the measurement of box-counting FD. The retinal vascular network FD can range from 1 to 2 . Lower FD values correspond to low complexity of vessel branching, while higher values correspond to greater complexity [10].

\section{Statistical analysis}

Continuous variables were tested for normality (Shapiro-Wilk test). Continuous variables that follow a normal distribution are expressed as mean \pm standard deviation (range) and those that follow a nonnormal distribution are expressed as median (interquartile range). Categorical variables are summarised in numbers and percentages $(n, \%)$. The Student's paired $t$ test was used to compare the differences between the scans by the two different operators. 
To analyse the repeatability and reproducibility of the retinal vascular network FD, we calculated the intraclass correlation coefficient (ICC) with $95 \%$ confidence intervals (Cls) using Shrout-Fleiss reliability notation. We followed Cicchetti's guidelines, which state that when the reliability coefficient is below 0.40 , the level of clinical significance is poor; when it is between 0.40 and 0.59 , the level of clinical significance is fair; when it is between 0.60 and 0.74 , the level of clinical significance is good; and when it is between 0.75 and 1.00 , the level of clinical significance is excellent [30]. Repeatability and reproducibility were also evaluated on the coefficient of variation (CV) calculated as standard deviation divided by the average of the measurements multiplied by 100 . A CV of less than $5 \%$ indicates good reproducibility. In addition, intra-observer repeatability and inter-observer reproducibility were evaluated by means of Bland-Altman analysis [31]. A $P$-value of less than 0.05 was considered statistically significant. All statistical analyses were performed using the statistical software SAS (version 9.4; SAS Institute, Inc., Cary, NC, USA).

\section{Results}

Overall, 43 eyes of 43 healthy volunteers were included in the analysis. The mean age of the participants was $30 \pm 6.2$ years (range, 23-52). Table 1 summarises the participants' baseline demographics and clinical characteristics. None of the participants demonstrated any eye disease on clinical examination or SS-OCTA.

Table 1

Baseline characteristics of the participants

\begin{tabular}{|lc|}
\hline \multicolumn{2}{|l|}{ Baseline characteristics } \\
\hline Age, years & $30 \pm 6.2$ \\
\hline Sex, female & $24(55.82)$ \\
\hline Study eye, right & $29(67.44)$ \\
\hline Axial length, $\mathrm{mm}$ & $23.7 \pm 1.4$ \\
\hline Body mass index, $\mathrm{kg} / \mathrm{m}^{2}$ & $21.88 \pm 2.1$ \\
\hline Systolic blood pressure, $\mathrm{mmHg}$ & $128.9 \pm 7.9$ \\
\hline Diastolic blood pressure, $\mathrm{mmHg}$ & $79.3 \pm 10.2$ \\
\hline Continuous variables are displayed as mean \pm standard deviation. \\
\hline Categorical variables are displayed as number (percentage). \\
\hline
\end{tabular}

The mean retinal vascular network FD in the superficial vascular layer was $1.694 \pm 0.010$ (range, 1.6561.709) for observer 1 and $1.693 \pm 0.014$ (range, 1.610-1.708) for observer 2 . The mean retinal vascular network FD in the deep vascular layer was $1.695 \pm 0.007$ (range, 1.662-1.704) and 1.693 \pm 0.011 (range, 1.624-1.705), for observers 1 and 2, respectively (Table 2). No significant difference was found between 
the two observers in terms of mean retinal vascular network FD for all measurements. Bland-Altman plots were used to demonstrate differences in overall mean FD measurements for the intra-observer repeatability and inter-observer reproducibility in the SCP and DCP (Figs. 2 and 3). The CV for each observer was less than $5 \%$, which indicates low variability and therefore good reproducibility (Table 2 ).

Table 2

Mean fractal dimension for the two observers

\begin{tabular}{|c|c|c|c|}
\hline & Observer 1 & Observer 2 & $\begin{array}{l}P \\
\text { value }\end{array}$ \\
\hline \multicolumn{4}{|c|}{ Average fractal dimension } \\
\hline & $\begin{array}{l}1.694 \pm \\
0.010\end{array}$ & $\begin{array}{l}1.693 \pm \\
0.014\end{array}$ & \multirow{2}{*}{$\begin{array}{l}0.59 \\
0.41\end{array}$} \\
\hline DCP & $\begin{array}{l}1.695 \pm \\
0.007\end{array}$ & $\begin{array}{l}1.693 \pm \\
0.011\end{array}$ & \\
\hline \multicolumn{4}{|c|}{ Coefficient of variation } \\
\hline SCP & 0.60 & 0.43 & \\
\hline DCP & 0.83 & 0.64 & \\
\hline $\begin{array}{l}\text { Conti } \\
\text { Coeff } \\
\text { SCP }\end{array}$ & $\begin{array}{l}\text { olayed as me } \\
\text { splayed as } p \\
\text { xus; DCP = }\end{array}$ & $\begin{array}{l}\text { standard de } \\
\text { ntage. } \\
\text { capillary ple }\end{array}$ & tion. \\
\hline
\end{tabular}

The intra-class correlation coefficients in the SCP and DCP were as follows. Intra-observer repeatability was $0.722(95 \% \mathrm{Cl}, 0.541-0.839)$ in the SCP and $0.828(95 \% \mathrm{Cl}, 0.705-0.903)$ in the DCP, deemed good and excellent, respectively [30]. Inter-observer reproducibility was $0.651(95 \% \mathrm{Cl}, 0.439-0.795)$ and 0.363 (95\% Cl, 0.073-0.596) in the SCP and DCP, respectively, deemed good and poor, respectively, according to Cicchetti [30].

\section{Discussion}

The present study aimed to describe FD metrics of the retinal vascular network using SS-OCTCA in healthy participants and to assess its repeatability and reproducibility in clinical practice. We used an SSOCTA device with automatic layer segmentation to achieve $12 \times 12-\mathrm{mm}$ images and then quantified the retinal vascular network FD of the SCP and DCP using independent software. The mean retinal vascular network FD was 1.693 in the superficial and 1.694 in the deep vascular layer in our healthy cohort. These results are in agreement with previous studies based on fundus photographs, which found a normal retinal vessel FD of $1.7[9,12]$. Although we did not use the same imaging device, our results were consistent since we acquired wide-field $12 \times 12-\mathrm{mm}$ images with a field of view of $48^{\circ}$, which allowed us to work on the fundus area close to that obtained with fundus photographs. Previous studies have 
investigated retinal vessel FD using SS-OCTA. Corvi et al. evaluated the retinal vascular network FD with seven different OCTA devices including the Cirrus Plex Elite 9000 (Carl Zeiss Meditec, Jena, Germany) using a $3 \times 3-\mathrm{mm}$ scan, and found a mean value of 1.68 and 1.69 in the superficial and deep vascular plexuses, respectively [18]. Although the acquisition size in our study was different, our results are in agreement. Hirano et al. quantified the retinal vascular network FD with the same SS-OCTA device and image size in healthy participants and in patients with diabetes [32]. They found a mean retinal vascular network FD of 1.627 and 1.612 in the superficial and deep capillary network in healthy eyes, respectively. However, to our knowledge, none have been interested in assessing the repeatability and reproducibility of this metrics. Furthermore, it should be noted that retinal vascular network FDs in the SCP and DCP were fairly similar. Few studies have compared the retinal vascular network FD in these two networks. It was reported that the patterns of these two networks are different, and that the central avascular zone is larger in the deep network compared with the superficial one [33]. FD evaluates the retinal vascular network as a whole, which may explain why there were only a few differences in the mean FD metrics between these two networks.

The retinal vascular network FD measurements showed good repeatability and reproducibility in our study. The measurements obtained by the same operator and those obtained by two operators were not statistically significantly different when the same protocol was used. Intra-observer repeatability was good in the SCP and excellent in the DCP. However, we found that the measurements of the retinal vascular network FD in the DCP did not have very good inter-observer reproducibility. This could be explained by the difficulty of removing projection artefacts of vessels from the superficial vascular network to the deep vascular network. It has already been shown that analysis of the superficial retinal plexus is more accurate than the deeper plexus because of projection artefacts [2]. Another explanation could be motion artefacts during OCTA acquisition. However, this did not occur between two acquisitions made by the same observer and therefore it could be related to changes in the head position between the two observers. Even small head movements or eye misposition can produce dramatic changes from one B-scan to another, although the SS-OCTA device eye-tracker tried to control for these artefacts.

The results regarding reproducibility in this study were only applicable to the SS-OCTA Cirrus Plex Elite 9000 (Carl Zeiss Meditec, Jena, Germany). Corvi et al. compared retinal vascular network FD measurements between seven different OCTA devices and found that the mean retinal vascular network FD in both the SCP and DCP were different between these instruments [18]. Differences were even found between devices using the same algorithm and segmentation limits. Thus, measurements between different OCTA devices were not interchangeable. Moreover, we only included healthy eyes in our study and, therefore, the automated segmentation was correct for all images. We should expect that in eyes with retinal disorders, automatic segmentation might compromise the repeatability of these measurements.

In previous studies, the retinal vascular network FD was considered an interesting biomarker for the study of retinal and cardiovascular diseases. These studies used methods based on the measurement of FD on fundus photographs $[12,27]$. With fundus photographs, it is impossible to distinguish the two vascular 
networks. Current advances in retinal imaging have allowed us to describe the retinal vascular network thoroughly. Furthermore, the swept-source technique has the advantage of being able to acquire larger images with better quality and contrast thus allowing us to study the vascular network as a whole [17]. Moreover, the benefit of using a $12 \times 12-\mathrm{mm}$ wide-field acquisition zone is to simultaneously visualise the macula region and the optic nerve head and to analyse both the macular network and radial peripapillary capillaries on the superficial and deep vascular plexus.

Fractal analysis can provide greater insight into the development of retinal vascular diseases. Recent studies have demonstrated the value of the retinal vascular network FD in clinical practice. A reduced retinal vascular network FD was found in all stages of diabetic retinopathy with SS-OCTA [32, 34]. Nevertheless, retinal vascular network FD analysis of the retinal network is a global measure of the blood vessel pattern; as such, it is not sensitive to minor alterations in a small region of the total pattern [10]. An earlier study explored the relationship between the retinal vascular network FD and neovascular agerelated macular degeneration (nAMD) [35]. The relationship between quantitative OCTA parameters in patients with active nAMD under treatment and those with remission nAMD was investigated. The retinal vascular network FD in the active nAMD group was significantly lower than that in the remission group (1.44 vs $1.50, P<0.001)$ [35]. This was explained by increased branching after arteriogenesis in the remission group. The retinal vascular network FD therefore seemed to be of interest for the study of retinal vascular diseases but also macular disorders. It would also be interesting to evaluate its contribution to the diagnosis of cardiovascular disorders. Combined with other quantitative parameters such as vascular density, vascular perfusion and the area of nonperfusion, the retinal vascular network FD offers the possibility of additional parameters to monitor retinal disease and to refine risk stratification.

The potential limitations of this study should be mentioned. First, it was a small-sample single-centre study, which may limit its external validity. Second, it was a self-report study; we did not carry out a medical assessment or blood test to eliminate vascular disease such as diabetes. Third, the age range was limited to individuals aged 24-52 years. Fourth, we had artefacts during OCTA image acquisition, including motion artefacts, as well as image treatment such as automated segmentation failure and projection artefacts [36].

In conclusion, the retinal vascular network FD provided new repeatable and reproducible quantitative data using SS-OCTA with healthy participants. The clinical relevance of these findings warrants further studies.

\section{Declarations}

\section{Ethics approval and consent to participate}

The study has been approved by the local Ethical Committee of Dijon University Hospital. Oral or written informed consent was obtained from all the participants. 


\section{Consent for publication}

All participants gave their informed consent and the study followed the tenets of the Declaration of Helsinki. Each participant gave their consent to publish the images and data from their ophthalmic examination (retinal image).

\section{Availability of data and materials}

The data that support the findings of this study are available from the Department of Ophthalmology of Dijon University Hospital but restrictions apply to the availability of these data, which were used under license for the current study, and so are not publicly available. Data are however available from the authors upon reasonable request to louis.arnould@chu-dijon.fr.

\section{Competing interests}

The authors declare that they have no non-financial competing interests

Disclosure: DH, None; LA, None; FB, Novartis (C), Thea (C); PHG, None; MS, None; AMB, Aerie (C), Allergan (C), Baush and Lomb (C), Santen Pharmaceutical (C), Théa (C); BA, None; CCG, Allergan (C), Bayer (C), Horus (C), Novartis (C), Roche (C), Théa (C)

\section{Funding}

No Funding

\section{Authors' contributions}

DH, analysis, interpretation of data, have drafted the work or substantively revised it; LA, analysis, interpretation of data, have drafted the work or substantively revised it; FB, revision of the draft; PHG, revision of the draft; $\mathrm{MS}$, revision of the draft; $\mathrm{AMB}$, revision of the draft; $\mathrm{BA}$, conception and design of the work; CCG, conception and revision of the work

Each authors have approved the submitted version and have agreed both to be personally accountable for the author's own contributions and to ensure that questions related to the accuracy or integrity of any part of the work, even ones in which the author was not personally involved, are appropriately investigated, resolved, and the resolution documented in the literature.

\section{Acknowledgements}

We thank Petra Eid for her support in collecting data.

\section{References}


1. Wong TY, Klein R, Klein BE et al (2001) Retinal microvascular abnormalities and their relationship with hypertension, cardiovascular disease, and mortality. Surv Ophthalmol 46:59-80

2. Patton N, Aslam T, Macgillivray T et al (2005) Retinal vascular image analysis as a potential screening tool for cerebrovascular disease: a rationale based on homology between cerebral and retinal microvasculatures. J Anat 206:319-348

3. Witt N, Wong TY, Hughes AD et al (2006) Abnormalities of retinal microvascular structure and risk of mortality from ischemic heart disease and stroke. Hypertension 47:975-981

4. Cheung N, Bluemke DA, Klein R et al (2007) Retinal arteriolar narrowing and left ventricular remodeling: the multi-ethnic study of atherosclerosis. J Am Coll Cardiol 50:48-55

5. Cheung N, Sharrett AR, Klein R et al (2007) Aortic distensibility and retinal arteriolar narrowing: the multi-ethnic study of atherosclerosis. Hypertension 50:617-622

6. Sasongko MB, Wong TY, Nguyen TT et al (2011) Retinal vascular tortuosity in persons with diabetes and diabetic retinopathy. Diabetologia 54:2409-2416

7. Seidelmann SB, Claggett B, Bravo PE et al (2016) Retinal vessel calibers in predicting long-term cardiovascular outcomes: the atherosclerosis risk in communities study. Circulation 134(18):13281338

8. Lemmens S, Devulder A, Van Keer K et al (2020) Systematic review on fractal dimension of the retinal vasculature in neurodegeneration and stroke: assessment of a potential biomarker. Front Neurosci14:16

9. Mainster MA (1990) The fractal properties of retinal vessels: embryological and clinical implications. Eye (Lond) 4:235-241

10. Masters BR (2004) Fractal analysis of the vascular tree in the human retina. Annu Rev Biomed Eng 6:427-452

11. Liew G, Wang JJ, Cheung $N$ et al (2008) The retinal vasculature as a fractal: methodology, reliability, and relationship to blood pressure. Ophthalmology 115:1951-1956

12. Avakian A, Kalina RE, Sage EH et al (2002) Fractal analysis of region-based vascular change in the normal and non-proliferative diabetic retina. Curr Eye Res 24:274-280

13. Orlando JI, Van Keer K, Barbosa Breda J et al (2017) Proliferative diabetic retinopathy characterization based on fractal features: evaluation on a publicly available dataset. Med Phys 44:6425-6434

14. Rosenfeld PJ, Durbin MK, Roisman L et al (2016) ZEISS angioplex spectral domain optical coherence tomography angiography: technical aspects. Dev Ophthalmol 56:18-29

15. Spaide RF, Fujimoto JG, Waheed NK et al (2018) Optical coherence tomography angiography. Prog Retin Eye Res 64:1-55

16. Spaide RF, Klancnik JM Jr, Cooney MJ (2015) Retinal vascular layers imaged by fluorescein angiography and optical coherence tomography angiography. JAMA Ophthalmol 133:45-50 
17. Potsaid B, Baumann B, Huang D et al (2010) Ultrahigh speed $1050 \mathrm{~nm}$ swept source/Fourier domain OCT retinal and anterior segment imaging at 100,000 to 400,000 axial scans per second. Opt Express 18:20029-20048

18. Corvi F, Pellegrini M, Erba S et al (2018) Reproducibility of vessel density, fractal dimension, and foveal avascular zone using 7 different optical coherence tomography angiography devices. Am J Ophthalmol 186:25-31

19. Lei J, Durbin MK, Shi Y et al (2017) Repeatability and reproducibility of superficial macular retinal vessel density measurements using optical coherence tomography angiography en face images. JAMA Ophthalmol 135:1092-1098

20. Lee MW, Kim KM, Lim HB et al (2019) Repeatability of vessel density measurements using optical coherence tomography angiography in retinal diseases. $\mathrm{Br} \mathrm{J}$ Ophthalmol 103:704-710

21. Xiao H, Liu X, Liao L et al (2020) Reproducibility of foveal avascular zone and superficial macular retinal vasculature measurements in healthy eyes determined by two different scanning protocols of optical coherence tomography angiography. Ophthalmic Res 63:244-251

22. Chen Q, Ma Q, Wu Ccet al (2017) Macular vascular fractal dimension in the deep capillary layer as an early indicator of microvascular loss for retinopathy in type 2 diabetic patients. Invest Ophthalmol Vis Sci 58:3785-37994

23. Bhardwaj S, Tsui E, Zahid S et al (2018) Value of fractal analysis of optical coherence tomography angiography in various stages of diabetic retinopathy. Retina 38:1816-23

24. Sng CCA, Wong WL, Cheung CY et al (2013) Retinal vascular fractal and blood pressure in a multiethnic population. J Hypertens 31:2036-2042

25. Arnould L, Binquet C, Guenancia C et al (2018) Association between the retinal vascular network with Singapore "I" Vessel Assessment (SIVA) software, cardiovascular history and risk factors in the elderly: the Montrachet study, population-based study. PLoS One 13:e0194694

26. Shi $C$, Chen $Y$, Kwapong WR et al (2020) Characterization by fractal dimension analysis of the retinal capillary network in parkinson disease. Retina 40:1483-1791

27. Talu S, Calugaru DM, Lupascu CA (2015) Characterisation of human non-proliferative diabetic retinopathy using the fractal analysis. Int $\mathrm{J}$ Ophthalmol 8:770-776

28. Kottner J, Kottner L, Brorson S et al (2011) Guidelines for reporting reliability and agreement studies (GRRAS) were proposed. J Clin Epidemiol 64:96-106

29. Landini G, Misson GP, Murray PI (1993) Fractal analysis of the normal human retinal fluorescein angiogram. Curr Eye Res 12:23-27

30. Cicchetti DV (1994) Guidelines, criteria, and rules of thumb for evaluating normed and standardized assessment instruments in psychology. Psychol Assess 6:284-290

31. Bland JM, Altman DG (1986) Statistical methods for assessing agreement between two methods of clinical measurement. Lancet 1:307-310 
32. Hirano T, Kitahara J, Toriyama Y et al (2019) Quantifying vascular density and morphology using different swept-source optical coherence tomography angiographic scan patterns in diabetic retinopathy. $\mathrm{Br} \mathrm{J}$ Ophthalmol 103:216-221

33. Bonnin S, Mané V, Couturier A et al (2015) New insight into the macular deep vascular plexus imaged by optical coherence tomography angiography. Retina 35:2347-2352

34. Karst SG, Heisler M, Lo J et al (2020) Evaluating signs of microangiopathy secondary to diabetes in different areas of the retina with swept source OCTA. Invest Ophthalmol Vis Sci 11;61:8

35. Coscas F, Cabral D, Pereira T et al (2018) Quantitative optical coherence tomography angiography biomarkers for neovascular age-related macular degeneration in remission. PLoS One 13:e0205513

36. Spaide RF, Fujimoto JG, Waheed NK (2015) Image artifacts in optical coherence tomography angiography. Retina 35:2163-2180

\section{Figures}



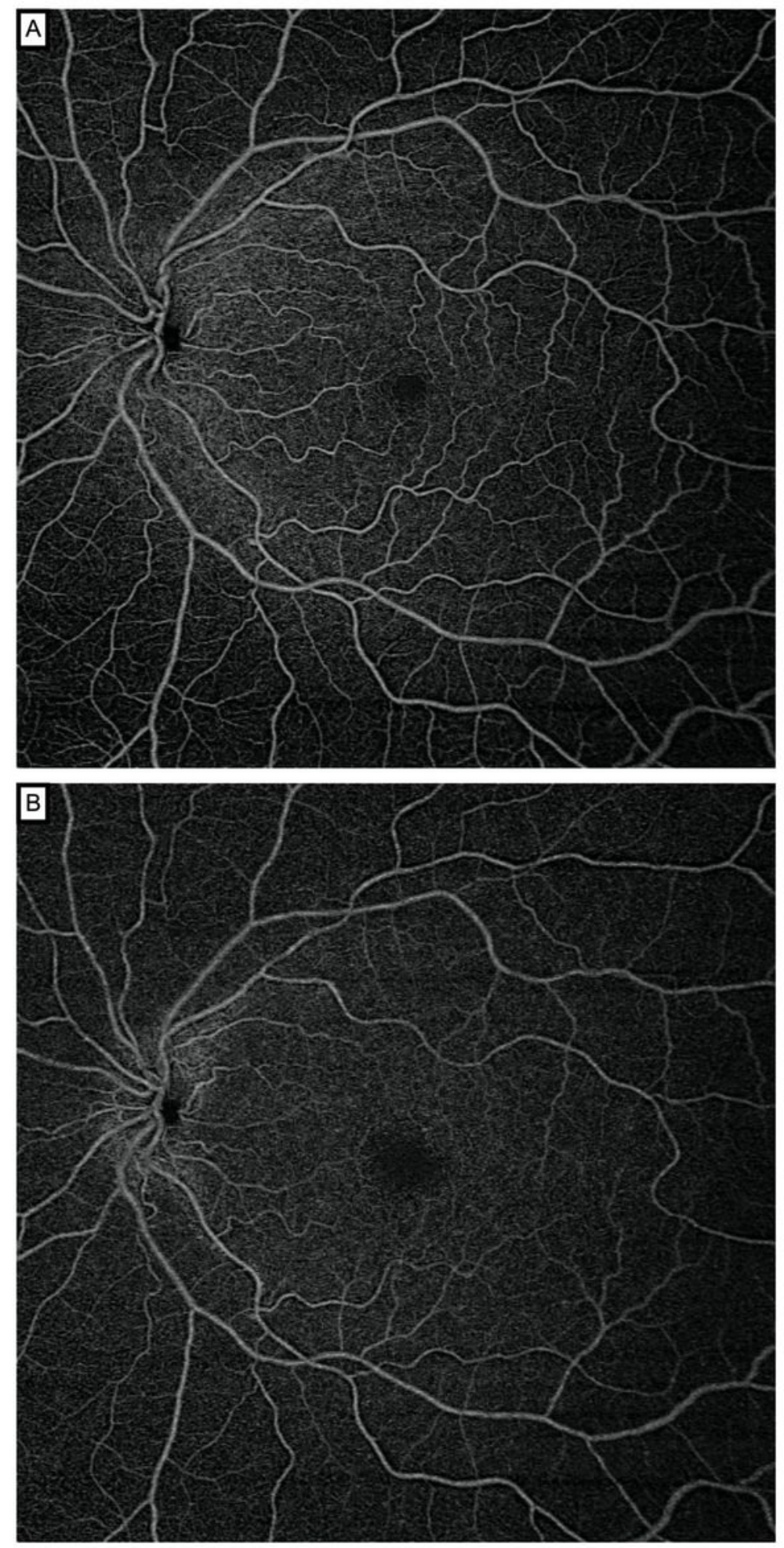

\section{Figure 1}

Swept-source optical coherence tomography angiography $12 \times 12-\mathrm{mm}$ en face images segmented through the superficial (A) and deep capillary plexus (B) of a healthy subject 
A

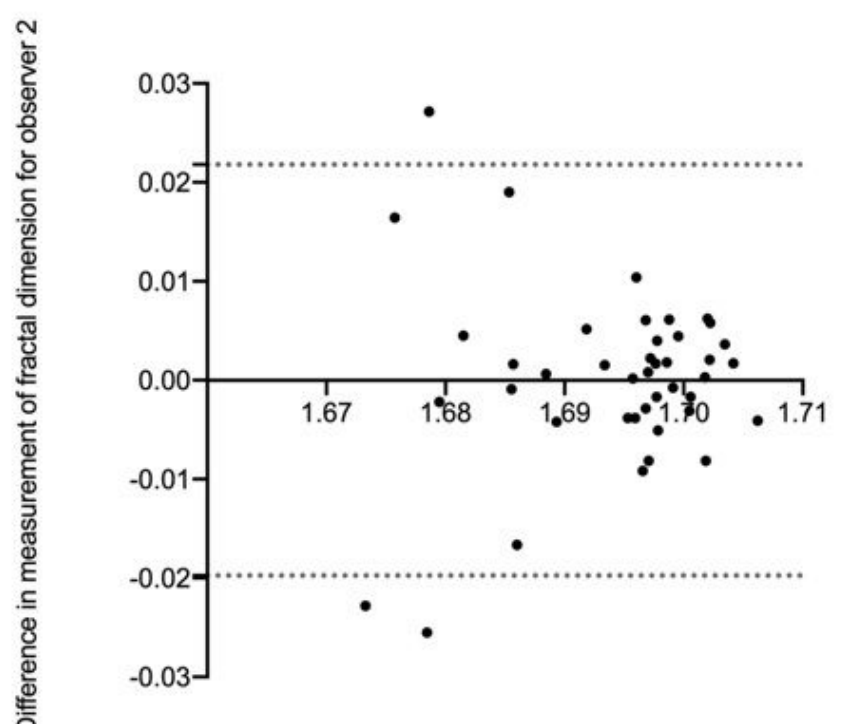

Mean of fractal dimension for observer 2

B

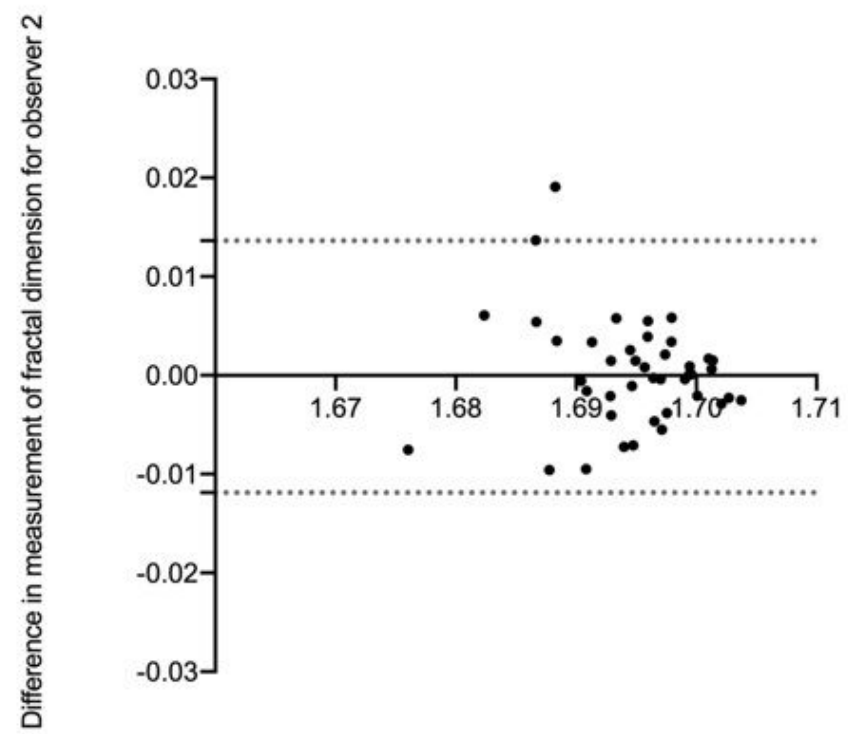

Mean of fractal dimension for observer 2

\section{Figure 2}

Bland-Altman plots showing intra-observer repeatability of retinal vascular network fractal dimension in the superficial (A) and deep capillary plexus (B) 


\section{A}

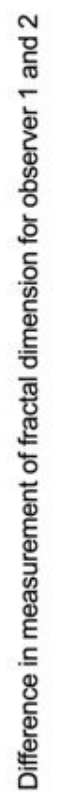

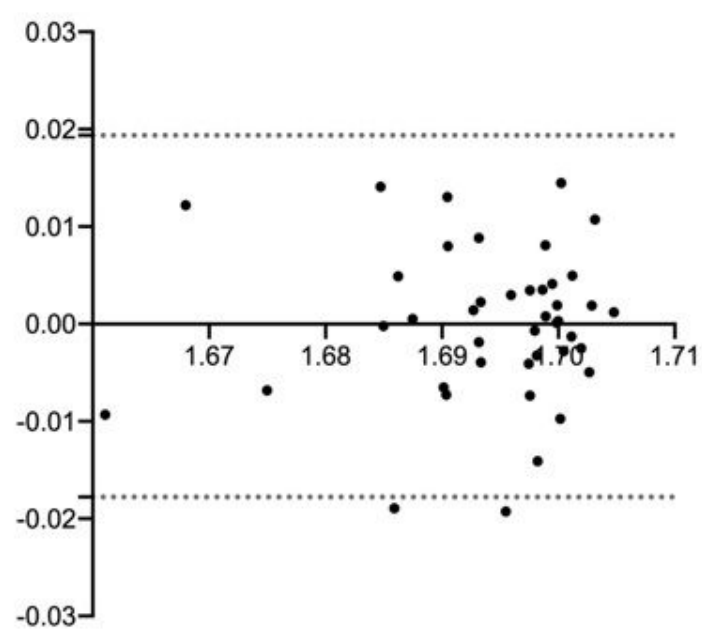

Mean of fractal dimension for observer 1 and 2

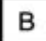

을

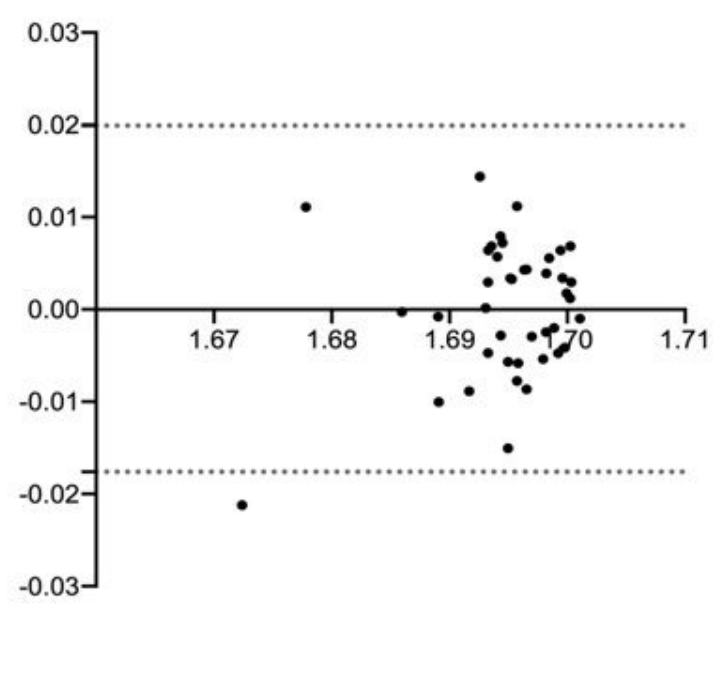

Mean of fractal dimension for observer 1 and 2

\section{Figure 3}

Bland-Altman plots showing inter-observer reproducibility of retinal vascular network fractal dimension in the superficial (A) and deep capillary plexus (B) 